\title{
BMJ Open Optimum duration of adjuvant trastuzumab in treatment of human epidermal growth factor receptor-2 positive early breast cancer: protocol for a network meta-analysis of randomised controlled trials
}

\author{
Qiancheng Hu, ${ }^{1}$ Xin Wang, ${ }^{1}$ Ye Chen, ${ }^{1}$ Xiaofen Li, ${ }^{1}$ Ting Luo, ${ }^{2}$ Dan Cao (D) ${ }^{1}$
}

To cite: Hu Q, Wang X, Chen Y, et al. Optimum duration of adjuvant trastuzumab in treatment of human epidermal growth factor receptor-2 positive early breast cancer: protocol for a network metaanalysis of randomised controlled trials. BMJ Open 2020;10:e035802. doi:10.1136/ bmjopen-2019-035802

- Prepublication history and additional material for this paper are available online. To view these files, please visit the journal online (http://dx.doi. org/10.1136/bmjopen-2019035802).

$\mathrm{QH}$ and $\mathrm{XW}$ are joint first authors.

Received 16 November 2019 Revised 05 November 2020 Accepted 10 November 2020

Check for updates

(C) Author(s) (or their employer(s)) 2020. Re-use permitted under CC BY-NC. No commercial re-use. See rights and permissions. Published by BMJ.

For numbered affiliations see end of article.

Correspondence to

Dr Dan Cao;

caodan316@163.com and

Dr Ting Luo;

tina621@163.com

\section{ABSTRACT}

Introduction Controversy regarding optimum duration of trastuzumab treatment remains in patients with human epidermal growth factor receptor-2 (HER2) positive early breast cancer. The objective of applying network metaanalysis (NMA) is to integrate existing evidence based on direct and indirect comparisons of efficacy and safety, and then to determine the duration of trastuzumab treatments with the greatest impact on therapeutic outcomes in HER2-positive early breast cancers.

Methods and analysis Electronic searching of trastuzumab treatments for early breast cancer by titles and abstracts will be conducted for the period from inception to 16 June 2019 in PubMed, Cochrane Library, Embase and ClinicalTrils.gov, as well as the annual meetings of San Antonio Breast Cancer Symposium (SABCS), European Society of Medical Oncology (ESM0) and American Society of Clinical Oncology (ASCO) online archives. The outcomes of interest are overall survival, disease-free survival, acceptability, cardiotoxicities and grade 3 to 4 non-haematological toxicities. Two independent reviewers will screen and extract eligible data based on the inclusion and exclusion criteria, and then assess the risk of bias and evidence quality of individual studies using Cochrane Collaboration's tool and Grades of Recommendation, Assessment, Development and Evaluation (GRADE). The heterogeneity, transitivity and inconsistency of NMA will be evaluated. In addition, we will perform subgroup and sensitivity analyses to assess the robustness and reliability of findings in our NMA.

Ethics and dissemination Ethics approval is not required for our NMA. Findings from our NMA will be submitted as peer-reviewed journal manuscripts and international conference reports.

Trial registration number CRD42019139109.

\section{INTRODUCTION}

Human epidermal growth factor receptor-2 (HER2) positive breast cancer accounts for approximately $20 \%$ to $25 \%$ of overall reported cases ${ }^{12}$ and is associated with poor
Strengths and limitations of this study

- Our objective of applying networkmeta-analysis (NMA) is to integrate existing evidence based on direct and indirect comparisons of efficacy and safety, and to determine the duration of trastuzumab treatments with the greatest impact on therapeutic outcomes in humanepidermal growth factor receptor-2 positive early breast cancers.

- Our study findings will help clinicians, patients and policymakers to reduce the uncertainty of escalating and de-escalating duration treatment and to select the optimum duration of trastuzumab treatment with highest efficacy and safety.

-We will perform subgroup and sensitivity analyses to assess the robustness and reliability of NMA results.

- Language bias is the potential limitation of our study as NMA will only include published studies in English.

prognosis. $^{3}{ }^{4}$ Trastuzumab, a monoclonal $^{2}$ antibody targeting the extracellular domain of the HER2 protein, is used for patients with HER2-positive early breast cancer. $^{5-7}$ Recently, targeted therapy using 1 year of trastuzumab has been proven to improve overall survival (OS) and disease-free survival (DFS) significantly in early HER2-positive breast cancer. ${ }^{8-11}$ Compared with treatment using chemotherapy only in early HER2positive breast cancer, treatment using adjuvant trastuzumab plus chemotherapy tends to reduce the risk of recurrence and death by one-third. ${ }^{12}$

However, the optimal duration of trastuzumab treatment has been an intense controversy and ongoing debate in terms of efficacy, toxicity, convenience and cost. ${ }^{13}$ High-quality randomised controlled trials 
(RCTs) have confirmed that multiple treatment durations of trastuzumab were effective for HER2-positive early breast cancers, but the relative efficacy and safety were not evaluated for all head-to-head trials. More specifically, the HERceptin Adjuvant (HERA) trial has confirmed that 24 months of adjuvant trastuzumab treatment, which was associated with a higher cost, inconvenience and cardiac toxicity $(7.3 \%$ vs $4.4 \%)$, would not improve DFS compared with 12 months of adjuvant therapy treatment (HR 1.02, 95\% CIs 0.89 to 1.17 ). ${ }^{14}$ While comparing to the 12 months of trastuzumab treatment, 6 months of trastuzumab treatment was noninferior and associated with decreased cardiac toxicity ( $8 \%$ vs $4 \%, \mathrm{p}<0.001)$ in the PERSEPHONE trial, but was not non-inferior in the PHARE and HORG trials. ${ }^{15-17}$ In contrast, the SOLD and Short-HER trials applying 9 weeks of trastuzumab was not non-inferior compared with the 12 months of trastuzumab, and a significant reduction in cardiac toxicity was observed in 9 weeks of trastuzumab. ${ }^{18} 19$

Direct comparison among preventive strategies was limited, as half of RCTs, including N9831, NSABP-B31, BCIRG 006 and FinHER trials, comparing active therapy to inactive interventions (eg, placebo). ${ }^{10} 1120$ Pivotal pairwise meta-analyses have been used to evaluate the efficacy and toxicity between shorter durations of trastuzumab and standard option directly. The analyses results suggested that 12 months of trastuzumab would still be the optimal treatment for early HER2-positive breast cancer, although with a significant increase in cardiac events. ${ }^{13}$ 21-24 The latest pairwise meta-analysis indicated that the use of trastuzumab in a 1-week cycle with anthracycline-taxane chemotherapy regimens simultaneously seemed to be the preferred option to optimise its efficacy and safety regardless of the duration of trastuzumab administration. ${ }^{12}$ However, the results were only from subgroup analysis, and the courses of trastuzumab administration were not only 12 months but also 9 weeks. Without direct comparison of RCTs, they did not contain 12 weeks, 6 months and 24 months of trastuzumab concurrently with chemotherapy compared with chemotherapy alone for early HER2-positive breast cancer in pairwise meta-analysis.

These intriguing results provoked an intense debate on consideration escalating and de-escalating duration treatment as new standard of care. Network meta-analysis (NMA) will provide indirect evaluations on the relative efficacy and toxicity of multiple durations of adjuvant trastuzumab therapies in HER2-positive early breast cancer. ${ }^{25}$ To address the aforementioned debate and determine the most appropriate treatment options, we will conduct NMA to integrate existing evidence available, based on direct and indirect comparisons of efficacy and safety, and to determine the duration of trastuzumab treatments (24 months vs 12 months vs 6 months vs 12 weeks vs 9 weeks vs placebo/observation/zero) with the greatest impact on therapeutic outcomes in HER2-positive early breast cancers.

\section{METHODS}

The results of our protocol will be evaluated in line with the Preferred Reporting Items for Systematic Reviews and Meta-Analyses Protocols (PRISMA-P).${ }^{26}$ Similarly, we will perform NMA in guidance of the PRISMA Extension Statement for Reporting of Systematic Reviews Incorporating Network Meta-Analyses of Healthcare Interventions. ${ }^{27}$ This project has been registered in PROSPERO (CRD42019139109).

\section{Search strategy}

Electronic searching by titles and abstracts of trastuzumab treatments for early breast cancers will be performed in PubMed, Cochrane Library, Embase (Ovid interface) and ClinicalTrials.gov, as well as the annual meetings of San Antonio Breast Cancer Symposium (SABCS) (20152019), European Society of Medical Oncology (ESMO) and American Society of Clinical Oncology (ASCO) online archives until 16 June 2019. Two reviewers who have been trained in data extraction will conduct search strategies independently. The same two authors will search reference lists manually from eligible reviews and relevant trials to identify additional potential papers. We will record the reasons of excluding the full text and generate a PRISMA flow diagram for the NMA. ${ }^{28}$

The terms used for literature searching will include the following domains of Medical Subject Heading (MeSH) terms: 'breast cancer', 'human epidermal growth factor receptor-2' and 'trastuzumab', according to Population Intervention Comparison Outcomes Study Design (PICOS) statement. MeSH and Subheadings will be combined with 'AND' or 'OR'. The complete search strategy is presented in online supplemental file 1 (see the online supplemental appendix 1).

We will perform a pilot test to evaluate inter-rater reliability and adjust each screening stage: title and abstract, followed by full-text screening. Two independent reviewers will screen the titles and abstracts of related studies based on inclusion and exclusion criteria. The eligible or potentially eligible trials will be evaluated by reading through the full texts when necessary. Moreover, disagreements in data extraction will be discussed with the help of the third reviewer.

\section{Eligibility criteria}

Trials will be eligible if they fulfil the following criteria: (1) Populations: patients with HER2-positive early breast cancer of any age or nationality were treated with trastuzumab treatments; (2) Interventions: any duration of trastuzumab treatments were given. We are also interested in the impact of placebo/observation/zero as adjuvant treatment; (3) Comparators: 12 months of trastuzumab treatment was compared with placebo/observation/zero, or other durations of adjuvant trastuzumab; (4) Outcomes: OS, DFS, acceptability, cardiotoxicities and grade 3 to 4 non-haematological toxicities; (5) Study design: RCTs that compared any two or more different arms of adjuvant trastuzumab in patients with HER2-positive early 
breast cancer; (6) Language and other limitations: We will include studies published in English regardless of publication status.

Studies not meeting the inclusion criteria will be excluded. The other excluding criteria are as follows: (1) Neoadjuvant and adjuvant treatment with trastuzumab biosimilars; (2) Palliative care with trastuzumab.

\section{Outcomes}

The outcomes of interest are OS (defined as the time from randomisation to death from any cause), DFS (defined as the time from randomisation to local, regional, distant relapse, contralateral breast cancer, second primary cancer or death from any cause, whichever occurred first), acceptability (defined as the proportion of patients who discontinued trastuzumab), cardiotoxicities and grade 3 to 4 non-haematological toxicities. The cardiac toxicity grading is used by the Common Terminology Criteria for Adverse Events of the National Cancer Institute. Cardiac toxicity is defined as an asymptomatic decline in left ventricular ejection fraction to $\leq 45 \%$, an absolute drop of $10 \%$ to $15 \%$ in follow-up echocardiography, symptomatic congestive heart failure (New York Heart Association (NYHA) class III/IV) or cardiac death. ${ }^{29}{ }^{30}$ We will calculate the relative effectiveness for each network comparison among all duration of treatments with trastuzumab. ${ }^{31}$

\section{Data extraction and management}

The management of literature searching records will be carried out in EndNote X7. A spreadsheet will be created in Microsoft Excel 2010 (Microsoft Corp, Redmond, Washington, www.microsoft.com) to collect outcomes of interest, such as study ID, first author, study design, recruitment time frame, detailed interventions, sample size and endpoints (OS, DFS, acceptability, cardiotoxicities and grade 3 to 4 non-haematological toxicities). We will contact corresponding authors and relevant pharmaceutical companies for further information if important data are not reported in articles. The most up-to-date data will be included if duplicate publications are identified.

\section{Bias risk}

The risk of bias of RCTs in the NMA will be evaluated by reviewer manager according to the following domains outlined in the Cochrane Collaboration's tool: random sequence generation, allocation concealment, blinding of participants and personnel, blinding of outcome assessment, incomplete outcome data, selective reporting and other bias. ${ }^{32}$ Two authors will review RCTs independently and report a high risk of bias as '-', a low risk of bias as '+' or an unclear risk of bias as '?'. Any disagreements in assessment of risk of bias will be resolved by discussion, or the help of the third reviewer if needed.

\section{Quality of evidence}

We will evaluate the quality of evidence of individual studies using Grades of Recommendation, Assessment, Development and Evaluation (GRADE), which is based on the following five domains: risk of bias, imprecision, inconsistency, indirectness and publication bias. ${ }^{33} 34$ The staging system categories for GRADE evidences are scored as high, moderate, low or very low quality. The initial confidence level for each RCT is set as high, but will be rated down based on the evaluation of the five domains. The strength of evidences will also be graded for the outcomes based on GRADE system in CINeMA. ${ }^{34}$

\section{Statistical analysis}

We will perform the traditional pairwise meta-analysis on direct comparisons based on two or more studies with Stata V.13.0 (StataCorp, College Station, Texas, USA). To compare eligible interventions directly and indirectly, NMA displaying outcomes of interest is planned using WinBUGS V.1.4.3 (MRC Biostatistics Unit, Cambridge, UK). Pooled HRs for OS and DFS with 95\% CIs will be calculated using both fixed-effects and random-effects models. Binary outcomes (acceptability, cardiotoxicities and grade 3 to 4 non-haematological toxicities) are expressed as ORs with 95\% CI. The results of comparative effectiveness and safety probability statements of intervention effects will be ranked; and rank plots across all outcomes will be generated. The interventions with surface under the cumulative ranking (SUCRA) in term of efficacy and safety will be evaluated to interpret relative effect of comparisons. We will compare the risk-benefit profile of all comparators in terms of efficacy and toxicity. A two-sided $p<0.05$ is considered statistically significant.

We will estimate the presence of heterogeneity based on the magnitude of $I^{2}$ estimated from pairwise meta-analysis models. The heterogeneity is considered as evidence of low if $I^{2}<25 \%$, as moderate if $25 \% \leq I^{2} \leq 50 \%$, and as high if $I^{2}>50 \%{ }^{35}$ The fixed-effects models will be used when the heterogeneity is low and moderate; otherwise, a random-effects models will be used. In addition, we will also evaluate the transitivity and inconsistency of NMA. The transitivity will be assessed by applying descriptive statistics for study types and demographic characteristics. Inconsistency will be assessed by comparing deviation information criteria statistics in the fitted consistency and inconsistency models. ${ }^{36}$ Global inconsistency between direct and indirect comparisons will also be evaluated by using a loop-specific method, if a loop connecting three or more arms exists. ${ }^{37}$

\section{Subgroup analysis}

We will explore whether specific duration of treatments with trastuzumab might be more appropriate for particular subtypes of breast cancer. We categorise breast cancer into the following groups when possible: Oestrogen Receptor (ER) positive, ER negative, node positive and node negative.

\section{Sensitivity analysis}

We will perform sensitivity analyses to assess the robustness and reliability of findings in our NMA. In order to check the impact of HER2 status on the results, the first sensitivity analysis will exclude patients with HER2 
negative after re-evaluating the HER2 status in the E2198 trial. $^{38}$ The second sensitivity analysis will restrict hormone receptor-positive to ER+ and progesteronereceptor (PR)+, ER+ and PR-, ER- and PR+. Lastly, the sensitivity analysis will classify patients as 1 to 3 and $\geq 4$ positive lymph nodes to specify the impact of the number of positive lymph nodes.

\section{DISCUSSION}

Despite trastuzumab being highly effective in treatment for HER2-positive early breast cancer, its substantial socioeconomic burden attracted the attention of governments, academic researchers, pharmaceutical companies and healthcare payers. With the consideration of balancing efficacy and cardiotoxicity, the 12-month and 6-month of trastuzumab treatments have received increasing interests. The requirement to balance efficacy and side effects (ie, cardiotoxicity) has led to raise interest in reducing trastuzumab duration from 12 months to 6 months. With the increase in rates of patients reporting 12-month trastuzumab-induced cardiotoxicity, most clinicians suggested that a drop to $83 \%$ 4-year DFS with 6 months trastuzumab would be also acceptable. ${ }^{39}$ This benefitrisk analysis will provide important information to help clinicians, patients and policymakers to decide optimum duration of adjuvant treatment with trastuzumab in their daily practice.

The 12 months of treatments with trastuzumab for most women with early HER2 positive breast cancer was a standard of care, but most crucial RCTs mainly focussed on patients with high-risk of recurrence and 1-year duration was chosen arbitrarily. In contrary, a particular subtype of patients might be appropriate for de-escalating duration of treatment, without compromising efficacy. BarrosoSousa and his colleagues deemed that de-escalating chemotherapy was a good option for older patients and those with stage I HER2-positive breast cancer. ${ }^{40}$ This study will explore whether de-escalating targeted therapy is another option for patients with particular subtypes (ER positive and node negative).

As far as we know, the results of system review will fill a pivotal knowledge gap of optimal duration of adjuvant trastuzumab in patients with early HER2-positive breast cancer. We hope the findings from this NMA will help clinicians, patients and policymakers to select optimal duration of adjuvant trastuzumab with the greatest value in HER2-positive early breast cancers. It will also provide a result that will engage patients and policymakers, and will contribute to the public debate on future policy options. Furthermore, under-recognised comparisons (eg, 6 months vs 9 weeks) may be identified by this Bayesian analysis to guide future research.

\section{Patient and public involvement}

The manuscript was developed without patient or public participation. Breast cancer patient organisations will participate in the discussion and dissemination of research results. A summary of the findings will be provided to the Chinese Society of Clinical Oncology (CSCO).

\section{Ethics and dissemination}

An ethics approval is not required for the NMA. Important modifications to the study protocol will be communicated to all members of the research team. The results will be disseminated through international conference reports and published in a peer-reviewed journal.

\section{Author affiliations}

${ }^{1}$ Department of Abdominal Oncology, Cancer Center, Sichuan University West China Hospital, Chengdu, Sichuan, China

${ }^{2}$ Breast Medical Oncology, Clinical Research Center for Breast, Sichuan University West China Hospital, Chengdu, Sichuan, China

Acknowledgements Thanks to Sun Feng and Wu Shanshan (PhD, Perking University School of Public Health) for providing assistance and contributing to statistical analysis. (https://class.dxy.cn/). Thanks to Lu Guan (PhD, Fisheries Oceans Canada) for providing assistance and contributing to revisions.

Contributors $\mathrm{QH}$ and $\mathrm{DC}$ conceptualised the network meta-analysis. $\mathrm{QH}$ and $\mathrm{XW}$ co-developed the search strategy. Both QH and XW were major contributors in writing the manuscript. The protocol was revised by DC, YC, XL and TL. DC and TL were serving as guarantor and corresponding author of this study. All authors approved the final manuscript and agreed to submit the protocol in the journal.

Funding The network meta-analysis was supported by the National Natural Science Foundation of China (Grant No.81773097).

Competing interests All authors have completed the ICMJE uniform disclosure form at http://www.icmje.org/coi_disclosure.pdf and stated that there is no organisation to support the submission; no organisation is interested in the submitted work; no other relationships or activities effect the submitted work.

Patient consent for publication Not required.

Provenance and peer review Not commissioned; externally peer reviewed.

Supplemental material This content has been supplied by the author(s). It has not been vetted by BMJ Publishing Group Limited (BMJ) and may not have been peer-reviewed. Any opinions or recommendations discussed are solely those of the author(s) and are not endorsed by BMJ. BMJ disclaims all liability and responsibility arising from any reliance placed on the content. Where the content includes any translated material, BMJ does not warrant the accuracy and reliability of the translations (including but not limited to local regulations, clinical guidelines, terminology, drug names and drug dosages), and is not responsible for any error and/or omissions arising from translation and adaptation or otherwise.

Open access This is an open access article distributed in accordance with the Creative Commons Attribution Non Commercial (CC BY-NC 4.0) license, which permits others to distribute, remix, adapt, build upon this work non-commercially, and license their derivative works on different terms, provided the original work is properly cited, appropriate credit is given, any changes made indicated, and the use is non-commercial. See: http://creativecommons.org/licenses/by-nc/4.0/.

ORCID iD

Dan Cao http://orcid.org/0000-0002-6709-4932

\section{REFERENCES}

1 Slamon DJ, Clark GM, Wong SG, et al. Human breast cancer: correlation of relapse and survival with amplification of the HER-2/ neu oncogene. Science 1987;235:177-82.

2 Slamon DJ, Godolphin W, Jones LA, et al. Studies of the HER-2/ neu proto-oncogene in human breast and ovarian cancer. Science 1989;244:707-12.

3 Curtis C, Shah SP, Chin S-F, et al. The genomic and transcriptomic architecture of 2,000 breast tumours reveals novel subgroups. Nature 2012;486:346-52.

4 Dawson S-J, Rueda OM, Aparicio S, et al. A new genome-driven integrated classification of breast cancer and its implications. Embo J 2013;32:617-28. 
5 Yeon $\mathrm{CH}$, Pegram MD. Anti-erbB-2 antibody trastuzumab in the treatment of HER2-amplified breast cancer. Invest New Drugs 2005;23:391-409.

6 Schaefer NG, Pestalozzi BC, Knuth A, et al. Potential use of humanized antibodies in the treatment of breast cancer. Expert Rev Anticancer Ther 2006;6:1065-74.

7 Tokunaga E, Oki E, Nishida K, et al. Trastuzumab and breast cancer: developments and current status. Int J Clin Oncol 2006;11:199-208.

8 Hortobagyi GN. Trastuzumab in the treatment of breast cancer. $N$ Engl J Med 2005;353:1734-6.

9 Piccart-Gebhart MJ, Procter M, Leyland-Jones B, et al. Trastuzumab after adjuvant chemotherapy in HER2-positive breast cancer. N Engl J Med 2005;353:1659-72.

10 Romond EH, Perez EA, Bryant J, et al. Trastuzumab plus adjuvant chemotherapy for operable HER2-positive breast cancer. N Engl J Med 2005;353:1673-84.

11 Slamon D, Eiermann W, Robert N, et al. Adjuvant trastuzumab in HER2-positive breast cancer. N Engl J Med 2011;365:1273-83.

12 Genuino AJ, Chaikledkaew U, The DO, et al. Adjuvant trastuzumab regimen for HER2-positive early-stage breast cancer: a systematic review and meta-analysis. Expert Rev Clin Pharmacol 2019;12:815-24.

13 Niraula S, Gyawali B. Optimal duration of adjuvant trastuzumab in treatment of early breast cancer: a meta-analysis of randomized controlled trials. Breast Cancer Res Treat 2019;173:103-9.

14 Cameron D, Piccart-Gebhart MJ, Gelber RD, et al. 11 years' followup of trastuzumab after adjuvant chemotherapy in HER2-positive early breast cancer: final analysis of the Herceptin adjuvant (HERA) trial. Lancet 2017;389:1195-205.

15 Earl HM, Hiller L, Vallier A-L, et al. 6 versus 12 months of adjuvant trastuzumab for HER2-positive early breast cancer (Persephone): 4-year disease-free survival results of a randomised phase 3 noninferiority trial. Lancet 2019;393:2599-612.

16 Pivot X, Romieu G, Debled M, et al. 6 months versus 12 months of adjuvant trastuzumab for patients with HER2-positive early breast cancer (PHARE): a randomised phase 3 trial. Lancet Oncol 2013:14:741-8

17 Mavroudis D, Saloustros E, Malamos N, et al. Six versus 12 months of adjuvant trastuzumab in combination with dose-dense chemotherapy for women with HER2-positive breast cancer: a multicenter randomized study by the Hellenic Oncology Research Group (HORG). Ann Oncol 2015;26:1333-40.

18 Joensuu $\mathrm{H}$, Fraser J, Wildiers $\mathrm{H}$, et al. Effect of adjuvant trastuzumab for a duration of 9 weeks vs 1 year with concomitant chemotherapy for early human epidermal growth factor receptor 2-positive breast cancer: the sold randomized clinical trial. JAMA Oncol 2018;4:1199-206.

19 Conte P, Frassoldati A, Bisagni G, et al. Nine weeks versus 1 year adjuvant trastuzumab in combination with chemotherapy: final results of the phase III randomized Short-HER studył. Ann Oncol 2018;29:2328-33.

20 Joensuu H, Bono P, Kataja V, et al. Fluorouracil, epirubicin, and cyclophosphamide with either docetaxel or vinorelbine, with or without trastuzumab, as adjuvant treatments of breast cancer: final results of the FinHer trial. J Clin Oncol 2009;27:5685-92.

21 Chen L, Zhou W, Hu X, et al. Short-Duration versus 1-year adjuvant trastuzumab in early HER2 positive breast cancer: a meta-analysis of randomized controlled trials. Cancer Treat Rev 2019;75:12-19.

22 Inno A, Barni S, Ghidini A, et al. One year versus a shorter duration of adjuvant trastuzumab for HER2-positive early breast cancer: a systematic review and meta-analysis. Breast Cancer Res Treat 2019;173:247-54.
23 Gyawali B, Niraula S. Duration of adjuvant trastuzumab in HER2 positive breast cancer: overall and disease free survival results from meta-analyses of randomized controlled trials. Cancer Treat Rev 2017;60:18-23

24 Goldvaser H, Korzets Y, Shepshelovich D, et al. Deescalating adjuvant trastuzumab in HER2-positive early-stage breast cancer: a systemic review and meta-analysis. JNCl Cancer Spectr 2019;3:pkz033

25 Clarke CS, Hunter RM, Shemilt I, et al. Multi-arm cost-effectiveness analysis (CEA) comparing different durations of adjuvant trastuzumab in early breast cancer, from the English NHS payer perspective. PLoS One 2017;12:e0172731.

26 Moher D, Shamseer L, Clarke M, et al. Preferred reporting items for systematic review and meta-analysis protocols (PRISMA-P) 2015 statement. Syst Rev 2015;4:1.

27 Hutton B, Salanti G, Caldwell DM, et al. The PRISMA extension statement for reporting of systematic reviews incorporating network meta-analyses of health care interventions: checklist and explanations. Ann Intern Med 2015;162:777-84.

28 Moher D, Liberati A, Tetzlaff J, et al. Preferred reporting items for systematic reviews and meta-analyses: the PRISMA statement. BMJ 2009;339:b2535.

29 Russell SD, Blackwell KL, Lawrence J, et al. Independent adjudication of symptomatic heart failure with the use of doxorubicin and cyclophosphamide followed by trastuzumab adjuvant therapy: a combined review of cardiac data from the National surgical adjuvant breast and bowel project B-31 and the North central cancer treatment group N9831 clinical trials. J Clin Oncol 2010;28:3416-21.

30 Yu AF, Singh JC, Wang R, et al. Cardiac safety of dual anti-HER2 therapy in the neoadjuvant setting for treatment of HER2-positive breast cancer. Oncologist 2017;22:642-7.

31 Salanti G. Indirect and mixed-treatment comparison, network, or multiple-treatments meta-analysis: many names, many benefits, many concerns for the next generation evidence synthesis tool. Res Synth Methods 2012;3:80-97.

32 Higgins JPT, Altman DG, Gøtzsche PC, et al. The Cochrane collaboration's tool for assessing risk of bias in randomised trials. BMJ 2011;343:d5928.

33 Guyatt GH, Oxman AD, Vist GE, et al. Grade: an emerging consensus on rating quality of evidence and strength of recommendations. $B M\lrcorner$ 2008;336:924-6.

34 Salanti G, Del Giovane C, Chaimani A, et al. Evaluating the quality of evidence from a network meta-analysis. PLoS One 2014;9:e99682.

35 Higgins JPT, Thompson SG, Deeks JJ, et al. Measuring inconsistency in meta-analyses. BMJ 2003;327:557-60.

36 Dias S, Welton NJ, Sutton AJ, et al. Evidence synthesis for decision making 4: inconsistency in networks of evidence based on randomized controlled trials. Med Decis Making 2013;33:641-56.

37 Veroniki AA, Vasiliadis HS, Higgins JPT, et al. Evaluation of inconsistency in networks of interventions. Int J Epidemiol 2013;42:332-45.

38 Schneider BP, O'Neill A, Shen F, et al. Pilot trial of paclitaxeltrastuzumab adjuvant therapy for early stage breast cancer: a trial of the ECOG-ACRIN cancer research Group (E2198). Br J Cancer 2015;113:1651-7.

39 Hiller L, Dunn JA, Loi S, et al. Adjuvant trastuzumab duration trials in HER2 positive breast cancer - what results would be practicechanging? Persephone investigator questionnaire prior to primary endpoint results. BMC Cancer 2018;18:391.

40 Barroso-Sousa R, Exman P, Tolaney SM. De-escalating treatment in the adjuvant setting in HER2-positive breast cancer. Future Oncol 2018;14:937-45. 